\title{
Metabolomics predicts the pharmacological profile of new psychoactive substances
}

\author{
Eulàlia Olesti1,2 (D), Ilario De Toma 2,3, Johannes G Ramaekers4, \\ Tibor M Brunt ${ }^{5,6}$, Marcel · lí Carbó ${ }^{2,7,8}$, Cristina Fernández-Avilés ${ }^{1}$, \\ Patricia Robledo1,2, Magí Farré9,10, Mara Dierssen 1,2,3,11,12, \\ Óscar J Pozo ${ }^{1}$ and Rafael de la Torre ${ }^{1,2,10,11}$
}

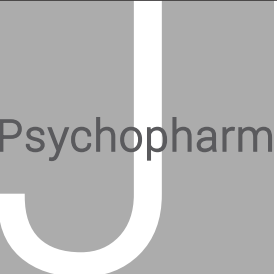

Journal of Psychopharmacology 
Current in silico screening approaches designed to mimic the interaction of drug-receptor could be proposed as an approach to describe a drug's mechanism of action (Kroeze et al., 2015; Strachan et al., 2006). However, the lack of information on the drug kinetics and metabolic disposition could bias the output obtained. In this context, we propose targeted metabolomics as a novel experimental approach for the high-throughput evaluation of emerging drugs. Nowadays, metabolomics is already applied as a tool to characterize the behaviour of diseases (Meinhardt et al., 2015) or used to discover pathology biomarkers (Näsström et al., 2014), among other applications (Wishart, 2016).

We hypothesized that exposure to NPS and classical drugs could produce drug-specific quantifiable changes in the targetmetabolomics profile formed by both monoamine neurotransmitters and steroid pathways, according to their mechanism of action. Indeed, by applying machine learning algorithms to the metabolomics fingerprints of traditional reference drugs (cocaine, methamphetamine, 3,4-methylenedioxyamphetamine (MDMA), $\Delta^{9}$-tetrahydrocannabinol ( $\Delta^{9}$-THC), and heroin) we could correctly predict the pharmacological profile of mephedrone and JWH-018 - assayed as models of NPS - with our constructed model.

\section{Materials and methods}

\section{Animals}

Male Wistar (Charles Rivers) rats aged 6 weeks and weighing 200-250 g were used in this study. They were housed in pairs in a room with controlled temperature $\left(22 \pm 1^{\circ} \mathrm{C}\right)$ and humidity $(55$ $\pm 10 \%$ ) under a $12 \mathrm{~h} \mathrm{light/dark} \mathrm{cycle} \mathrm{(on} \mathrm{at} \mathrm{8:00} \mathrm{and} \mathrm{off} \mathrm{at}$ 20:00). Food and water were available ad libitum. Animals were handled and habituated to the experimental room 1 week prior to testing. All animal procedures were conducted in accordance with the standard ethical guidelines (European Communities Directive 86/60-EEC) and approved by the local ethical committee (Comitè Ètic d'Experimentació Animal of the Barcelona Biomedical Research Park; CEEA-PRBB).

\section{Drugs}

MDMA and 4-methylmethcathinone (mephedrone) were obtained through the Division of Narcotic Drugs and Psychotropic Substances of the Spanish Medicines Agency (AEMPS). Methamphetamine, heroin and cocaine were obtained from Sigma-Aldrich(SaintLouis, MO,USA). $\Delta^{9}$-Tetrahydrocannabinol $\left(\Delta^{9}\right.$-THC) was obtained from THC Pharm GmbH (Frankfurt, Germany). JWH-018 was purchased from LGC Standards (Lancashire, UK). MDMA (15 mg/kg), cocaine (20 mg/kg), methamphetamine $(15 \mathrm{mg} / \mathrm{kg})$, heroin $(30 \mathrm{mg} / \mathrm{kg})$ and mephedrone $(30 \mathrm{mg} / \mathrm{kg})$ were diluted in saline $(0.9 \%$ bacteriostatic sodium chloride). $\Delta^{9}-\mathrm{THC}(15 \mathrm{mg} / \mathrm{kg})$ and JWH-018 $(10 \mathrm{mg} / \mathrm{kg})$ were diluted in vehicle (5\% ethanol, $5 \%$ cremophor, $90 \%$ saline). All drugs were administered intraperitoneally at a volume of $1 \mathrm{ml} / \mathrm{kg}$, except for the ethanol-chremophor vehicle, $\Delta^{9}$-THC and JWH-018, which was administered at $5 \mathrm{~mL} / \mathrm{kg}$.

Tryptophan (Trp), 5-hydroxytryptamine, serotonin (5-HT), 5-hydroxyindole acetic acid (5-HIAA), phenylalanine (Phe), tyrosine (Tyr), dopamine (DA), 3-methoxytyramine (3-MT), 3,4-dihydroxyphenylacetic acid (Dopac), homovanillic acid (HVA), norepinephrine (NE), epinephrine (E), metanephrine (ME) and normetanephrine (NME) were obtained from Sigma-Aldrich (Saint Louis, MO, USA). Progesterone (Prog), deoxycorticosterone (DOC), corticosterone (B), 11-dehydrocorticosterone (A), androstenedione (AED) and testosterone (T) were also purchased from Sigma-Aldrich (Saint Louis, MO, USA). Trp- $\mathrm{d}_{5}, \quad$ 5-HIAA- $\mathrm{d}_{4}, \quad$ Tyr- $\mathrm{d}_{4}, \quad 3-\mathrm{MT}_{-} \mathrm{d}_{4}$, Dopac- $\mathrm{d}_{3}$, ${ }^{13} \mathrm{C}-\mathrm{HVA}-\mathrm{d}_{3}, \mathrm{NE}-\mathrm{d}_{3}$ and $\mathrm{MN}-\mathrm{d}_{3}$ were purchased from Alsachim (Illkirch-Graffenstaden, France). 5-HT- $\mathrm{d}_{4}$, Phe- $\mathrm{d}_{5}$, DA- $\mathrm{d}_{4}$ and Cortisone- $\mathrm{d}_{8}$ were obtained from Toronto Research Chemicals (Toronto, Canada). Cortisol- $\mathrm{d}_{4}$ and testosterone- $\mathrm{d}_{3}$ were obtained from Sigma-Aldrich (St Louis, MO, USA) and NMI (Pymble, Australia), respectively. Methanol, acetonitrile, acetone, ethyl acetate and sodium chloride were purchased from Merck (Darmstadt, Germany). Ultra-pure water was obtained with a Milli-Q system. Dansyl chloride was obtained from SigmaAldrich (Missouri, USA). The $\beta$-glucuronidase preparation (type K12 from Escherichia coli) was purchased from Roche Diagnostics GmbH (Mannheim, Germany).

\section{Experimental procedure}

Two different experimental designs were followed. First, to determine rapid metabolomics changes in brain tissue, in experiment 1 (Supplementary Figure 1(a)) rats were habituated to the metabolic cages for $2 \mathrm{~h}$. The next day they received a single administration of drug $(n=6)$ or vehicle $(n=6)$ and were placed in the metabolic cages for $1 \mathrm{~h}$. Subsequently, rats were anaesthetised (with isoflurane) to collect blood samples by intra-cardiac puncture, followed by euthanasia and brain extraction. The prefrontal cortex (PFC), the cerebellum (Cer), the hippocampus (HC) and the striatum (St) were dissected, placed in liquid nitrogen and later stored at $-80^{\circ} \mathrm{C}$ until processing. Second, to determine sustained metabolomics changes in plasma, urine and brain tissue, in experiment 2 (Supplementary Figure 1(b)) rats were habituated to the metabolic cages for $2 \mathrm{~h}$. The next day they were placed in the metabolic cages for $4 \mathrm{~h}$, and urine was collected for basal measurements. The following day, they received a single administration of the vehicle and were again placed in the metabolic cages for $4 \mathrm{~h}$. Urine was collected once more, which served as a control for the intraperitoneal injection. On day 4, rats received a single administration of drug $(n=6)$ or vehicle $(n=6)$ and were placed in the metabolic cages for $4 \mathrm{~h}$. Then, urine was collected and rats were anaesthetised to collect blood samples by intra-cardiac puncture, followed by brain extraction and harvesting of brain areas, as described above. The circadian rhythms variations were minimized by collecting all the samples at the same time-period in all treated groups (with drugs or saline) in all conditions ( $1 \mathrm{~h}$ and $4 \mathrm{~h}$ post drug/ vehicle administration).

\section{Sample preparation}

The brain areas were homogenized with $1 \mathrm{~mL}$ tissue grinder dounce (Wheaton, USA). The tissue was processed in $400 \mu \mathrm{L}$ of formic acid at $0.1 \%$, and protein precipitation was obtained with $800 \mu \mathrm{L}$ of acetonitrile. The mixture was centrifuged $(10 \mathrm{~min}$, $\left.13.000 \mathrm{rpm}, 4^{\circ} \mathrm{C}\right)$ and the supernatant was kept at $-20^{\circ} \mathrm{C}$ until analysis. Blood was preserved at $4^{\circ} \mathrm{C}$ during the experimental 
collection, and plasma was separated by centrifugation at $4^{\circ} \mathrm{C}$ and $1700 \mathrm{~g}$ for $15 \mathrm{~min}$.

\section{Monoamine neurotransmitter quantification}

For the proper multi-compound quantification of the endogenous monoamine metabolic pathway, a validated LC-MS/MS method was used (Marcos et al., 2016) with a dansyl chloride derivatization-validated methodology (Cai et al., 2010; Lu et al., 2016). Monoamines were quantified in different brain areas and in urine (Supplementary Figure 1(c) to (e)). For brain areas, calibration range varied from 10 to $1000 \mathrm{ng} / \mathrm{mL}$ and from 50 to $5000 \mathrm{ng} / \mathrm{mL}$ in urine.

\section{Steroid hormone quantification}

Steroid hormones were quantified in brain areas and plasma through a validated methodology (Marcos et al., 2014). Steroid hormones were quantified in brain areas and in plasma (see Supplementary Figure 1(f)). For brain areas and plasma, the calibration range varied from 2 to $1000 \mathrm{ng} / \mathrm{mL}$.

\section{Instrumentation}

An Acquity UPLC instrument (Waters Associates, Milford, MA, USA) was used for the liquid chromatographic (LC) separation. The system was supplied with an Acquity BEH C18 column (100 mm, $2.1 \mathrm{~mm}$ i.d., $1.7 \mu \mathrm{m})$ (Waters Associates), at $55^{\circ} \mathrm{C}$ and with a constant flow rate of $300 \mu \mathrm{L} / \mathrm{min}$. Methanol and water, both containing formic acid $(0.01 \% \mathrm{v} / \mathrm{v})$ and ammonium formate $(1 \mathrm{mM})$ were used as mobile phase solvents.

The chromatographic system was coupled to a triple quadrupole (Quattro Premier Waters) mass spectrometer coupled with an orthogonal Z-spray-electrospray interface (ESI). The nebulizing gas used was nitrogen. The nitrogen desolvation temperature was set to $450^{\circ} \mathrm{C}$, and the source temperature was $120^{\circ} \mathrm{C}$. The cone gas flow was set to $50 \mathrm{~L} / \mathrm{min}$, and the desolvation gas flow was set to approximately $1200 \mathrm{~L} / \mathrm{h}$. The ionization was in positive mode with a capillary voltage of $3 \mathrm{kV}$. Argon was used as a collision gas with a constant flow of $0.21 \mathrm{~mL} / \mathrm{min}$.

The multi-analytes studied were determined by Selected Reaction Monitoring. For each analyte, at least two transitions, optimized in cone voltage and collision energy, were acquired. The most specific transition with the lowest noise ratio signal was selected for quantitative purposes. MassLynx software (Waters Associates) was used for peak integration and data management.

\section{Metabolomics data analysis}

Through the MassLynx software we obtained the concentrations for each sample analyzed (with calibration curves). In brain areas, concentration was corrected with the weighting area. In urine samples, the correction was normalized by the excreted volume.

A heatmap was drawn to show the dynamics of the metabolites (Figure 1). It permitted the representation of a visual increase/decrease of non-altered marker relation. For the construction of the heatmap, ratio values were calculated in all biological matrices. Ratios were obtained by dividing the metabolite marker concentration after drug/vehicle administration by the median values of the vehicle administration for each sample. These ratios were calculated in plasma and brain areas (PFC, Cer, HC and St). In urine, the ratio was calculated by dividing metabolites recovered $0-4 \mathrm{~h}$ after drug administration with metabolites recovered from the median of vehicle administration. All ratios were represented in the heatmap, and the following code was applied to the ratio values: 0.2 (blue), 1 (yellow) and 5 (red), in a gradual manner. Intense red had a closer ratio value to 5 and thus a greater increment of the marker in comparison with the vehicle administration. In contrast, intense blue reflected the decrease of a marker in comparison with its vehicle. Yellow values showed no-change against the vehicle administration. In the heat maps for the three neurotransmitter pathways there are four columns corresponding to the four brain areas evaluated and an additional column only filled at $4 \mathrm{~h}$ corresponding to metabolomics changes in urine $(0-4 \mathrm{~h})$. Regarding steroids, the additional column for brain results corresponds to plasma concentrations ( $1 \mathrm{~h}$ and $4 \mathrm{~h}$ post drug administration).

The metabolomics fingerprints in urine were determined by calculating the ratio between basal and drug administration values within subjects, and in plasma and brain tissue by calculating the ratio between vehicle and drug administration values between subjects. The data were analyzed with the non-parametric MannWhitney test, since it was not normally distributed according to the Kolmogorov-Smirnov test. Significant differences were considered when $p$-values were $<0.05$.

\section{Data preparation and principal component analysis (PCA)}

Before performing the PCA, missing values were imputed using the missMDA R package. Then testing from 0 to 15 numbers of components (using the estim_ncpPCA function) we estimated the number of dimensions for the PCA by cross validation. The number of components that led to the smallest mean square error of prediction was then used to predict the missing entries by using the imputePCA function with a regularized iterative PCA algorithm. The negative imputed values were replaced by 0 and a pseudo-value of 0.001 was added to each cell to be able to take the logarithm for those variables whose normality improved upon the log-transformation (as measured by a higher $p$-value from the Shapiro-Wilk test of normality upon transformation). For the PCA, we used R prcomp function, scaling and centring the variables to have the unit variance and zero centred mean before the analysis.

\section{Classification model: random forest}

The classification model was built using the random forest algorithm (method "rf" in the train function in the caret $\mathrm{R}$ package) using the targeted metabolomics data of the canonical drugs. For accuracy estimation we used the leave-one-out cross validation (LOOCV). In the LOOCV method, we built a new model leaving one of the samples out. This new model was used to predict the left-out instance. This was repeated for all data instances. Accuracy was the fraction of correct predictions. Then, variable importance was calculated with the varImp function of the caret package. 


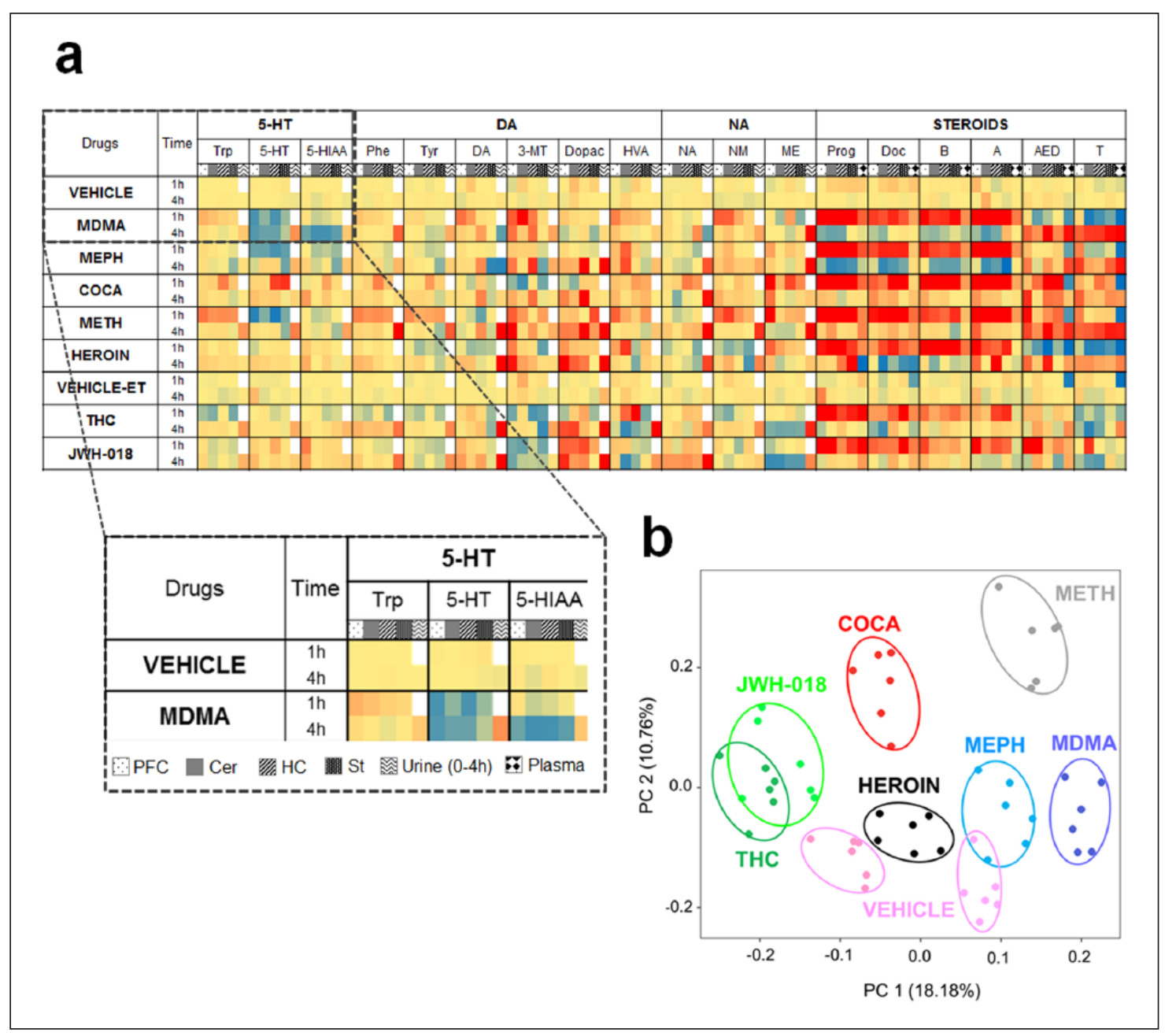

Figure 1. Heat map of the targeted metabolomics alterations of different classical and NPS drugs in comparison with the vehicle administration and principal component analysis representation with all the metabolomics data. (a) Changes in the metabolomics profile monitored in four brain areas (PFC, Cer, $\mathrm{HC}$ and St) and plasma (only steroids) at 1 and $4 \mathrm{~h}$ after drug administration and in urine (0-4 h urine, only monoamines). For every pathway evaluated, rows display mean ratio values $(n=6)$. For the construction of the heatmap, the following code was applied to the ratio values: 0.2 (blue, decrease of a marker), 1 (yellow) and 5 (red, increase of a marker in comparison with vehicle), in a gradual manner. The yellow values show no-change against the vehicle administration. (b) The bi-plot shows the principal component 1 on the $x$-axis and the principal component 2 on the $y$-axis. The percentage of variance explained by the two components is indicated in brackets. Each dot corresponds to an individual sample.

The metabolomics data from rats treated with NPS were given as an input to the predict function that calculated for each sample the votes of the trees in the ensemble. Results were shown by heatmap plotted with the gplot package. The code is available on Bitbucket (https://bitbucket.org/ilario_de_toma/ NPSs).

To check for overfitting, we built 168 different models adding a metabolite one by one, from the most important to the least important. We therefore plotted the accuracy as calculated by the LOOCV in function of the number of variables included. In a similar manner, for each of these 168 models we calculated their ability to correctly predict NPS. Three scores were employed:

- Score calculated by adding $1 / 3$ point for each well-predicted NPS

- Sum of the probabilities of prediction for mephedrone as MDMA, and JWH-018 as THC
- The sum of the differences, in the case of correct prediction, between the first prediction and the second (the higher the value, the greater the certainty and reproducibility of the prediction)

We also quantile normalized these scores to obtain a single one.

\section{Results}

\section{Identification of drug-specific metabolomics fingerprints}

We classified a number of traditional drugs of abuse (cocaine, methamphetamine, MDMA, heroin, and $\Delta^{9}$-tetrahydrocannabinol $\left(\Delta^{9}\right.$-THC) $)$ and two NPS (mephedrone and JWH-018) according to their metabolomics profile. Our metabolomics study included 168 markers: 18 metabolites from the serotonergic, dopaminergic 
and noradrenergic pathways, corticosteroids, and sexual hormones that were tested at two different time points in six biological matrices (hippocampus, prefrontal cortex, cerebellum, striatum, plasma and urine) (Supplementary Figure 1). The metabolomics fingerprint was obtained for all drugs evaluated by determining the changes in concentrations of selected precursors and metabolites of neurotransmitters and steroids in brain tissue, urine and plasma at 1 and $4 \mathrm{~h}$ following drug administration (Supplementary Tables 1-7). These fingerprints are represented in Figure 1(a) as a heatmap of increases or decreases of the markers observed for each drug versus vehicle.

The metabolomics fingerprints of psychostimulant drugs, such as MDMA, mephedrone and methamphetamine, showed a depleting effect on serotonin brain concentrations $1 \mathrm{~h}$ post administration. Of these, MDMA induced the most generalized effect on the serotonergic system, as changes were observed in all brain regions for serotonin and its main metabolite, 5-HIAA. Effects were maintained $4 \mathrm{~h}$ later, particularly in the striatum.

All drugs boosted to some extent the turnover of the dopaminergic system, although such effects were more pronounced for methamphetamine, heroin and particularly JWH-018. In contrast, $\Delta^{9}$-THC and JWH-018 presented lower recovery of 3-methoxytyramine (Bloomfield et al., 2016). No major effects were observed for heroin, THC and JWH-018 on norepinephrine release in the brain, while psychostimulant drugs increased norepinephrine levels and the turnover of its metabolites in all brain areas in the first hour post administration. Additionally, the recovery of normetanephrine and metanephrine, two additional COMT metabolites of norepinephrine and adrenaline, respectively, was much lower for $\Delta^{9}$-THC and JWH-018 than for the other drugs of abuse.

In general, psychostimulant drugs such as cocaine, methamphetamine, MDMA, and mephedrone - considered chemical stressors - boosted, with different degrees of intensity, the release of progesterone and corticosteroids in the first hour, and this effect was observed in androgens at $4 \mathrm{~h}$ post administration. In the case of heroin, the metabolomics profile was quite similar, while for THC and JWH-018 we observed only minor changes in testosterone concentrations.

Changes in plasma metabolomics of steroid compounds mimicked those observed in the brain in our experimental conditions. In contrast, metabolomics in rat urine was a poor predictor of the changes occurring in the brain for all neurotransmitter systems and steroids.

PCA of metabolomics fingerprints (Figure 1(b)) showed that rats treated with the same substance clustered together, while those that received different drugs were clearly separated when plotting the first two principal components. Moreover, the variance in the target metabolome was maximal among drugs with different pharmacological profile and minimal among those with a similar profile. Interestingly, mephedrone clustered close to MDMA, while JWH-018 was near THC.

\section{A predictive classification of NPS according to its metabolomics fingerprint}

As our PCA showed that rats treated with drugs clustered together (Figure 1(b)), our next goal was to build a predictive model that correctly classified each classical drug according to its targeted metabolic profile. For this purpose, we trained a model using the machine learning random forest approach (Breiman, 2001) with the individual data of each rat receiving a classical drug $(n=6$ per group). This model had an accuracy of $100 \%$ as assessed by LOOCV (see Methods).

We then tested our model with metabolomics data from rats that were treated with mephedrone and JWH-018 in order to investigate their similarity with classical drugs. As expected, our model predicted mephedrone as being comparable to MDMA in all samples. Indeed, both psychostimulants share pharmacological properties: mephedrone interacts with 5-HT and DA transporters in a similar manner to MDMA (Baumann et al., 2012; Martínez-Clemente et al., 2012; Simmler et al., 2013). Our model also correctly predicted JWH-018 to be a $\Delta^{9}$-THC-like compound based on its metabolomics profile, which is in accordance with its equivalent interaction with the cannabinoid receptors (Maldonado et al., 2011). Such results demonstrate that targeted metabolomics can be used to predict the pharmacological profile of our two test NPS (Figure 2(a)). Furthermore, in order to optimize the cost-effectiveness of our methodology we constructed the prediction model only with data from the six animals euthanized at $1 \mathrm{~h}$ post drug administration, and the result was again successfully achieved (Figure 2(b)).

In order to avoid possible issues of overfitting, we subsequently investigated whether the number of relevant variables could be reduced without losing accuracy. Several reduced models were constructed adding one by one the most relevant metabolites (in order of their importance in the random forest model). A stable $100 \%$ accuracy was reached when the 20 major metabolites were included. In addition, by incorporating the first 66 major metabolites, we correctly predicted all mephedrone- and JWH-018-treated samples as being similar to MDMA and THC, respectively (Supplementary Figure 2). When evaluating these 66 metabolites, they belonged to serotonin (15 metabolites), dopamine (21), norepinephrine (15), and steroid (15) pathways, thus being almost equally as important for prediction. Brain metabolites were the most important, so that metabolites from the hippocampus (17 metabolites), the prefrontal cortex (11) and the striatum (17) accounted for around $70 \%$ of the 66 "predictive" metabolites. Finally, we observed that metabolites measured at $1 \mathrm{~h}$ (39 metabolites) and also at $4 \mathrm{~h}$ (27 metabolites) post drug administration were equally relevant for model fitting (Supplementary Figure 2, Supplementary Figure 3, and Supplementary Table 8).

\section{Discussion}

We have developed a new pharmacological profiling model that employs drug-specific metabolomics fingerprints based on the quantification of monoamine neurotransmitters and steroid hormones in rats to predict the similarity of NPS with specific drug categories.

The model was trained only with data from the brains of rats administered with classical drugs since novel NPS are intended to mimic their effects. Our findings are consistent with previous work (Fleckenstein et al., 1997; Stone et al., 1986) as it was observed that drugs such as methamphetamine and MDMA, described as decreasing the enzymatic activity of tryptophan hydroxylase, increased its precursor (Trp) and decreased its product (5-HT) $1 \mathrm{~h}$ post drug administration 


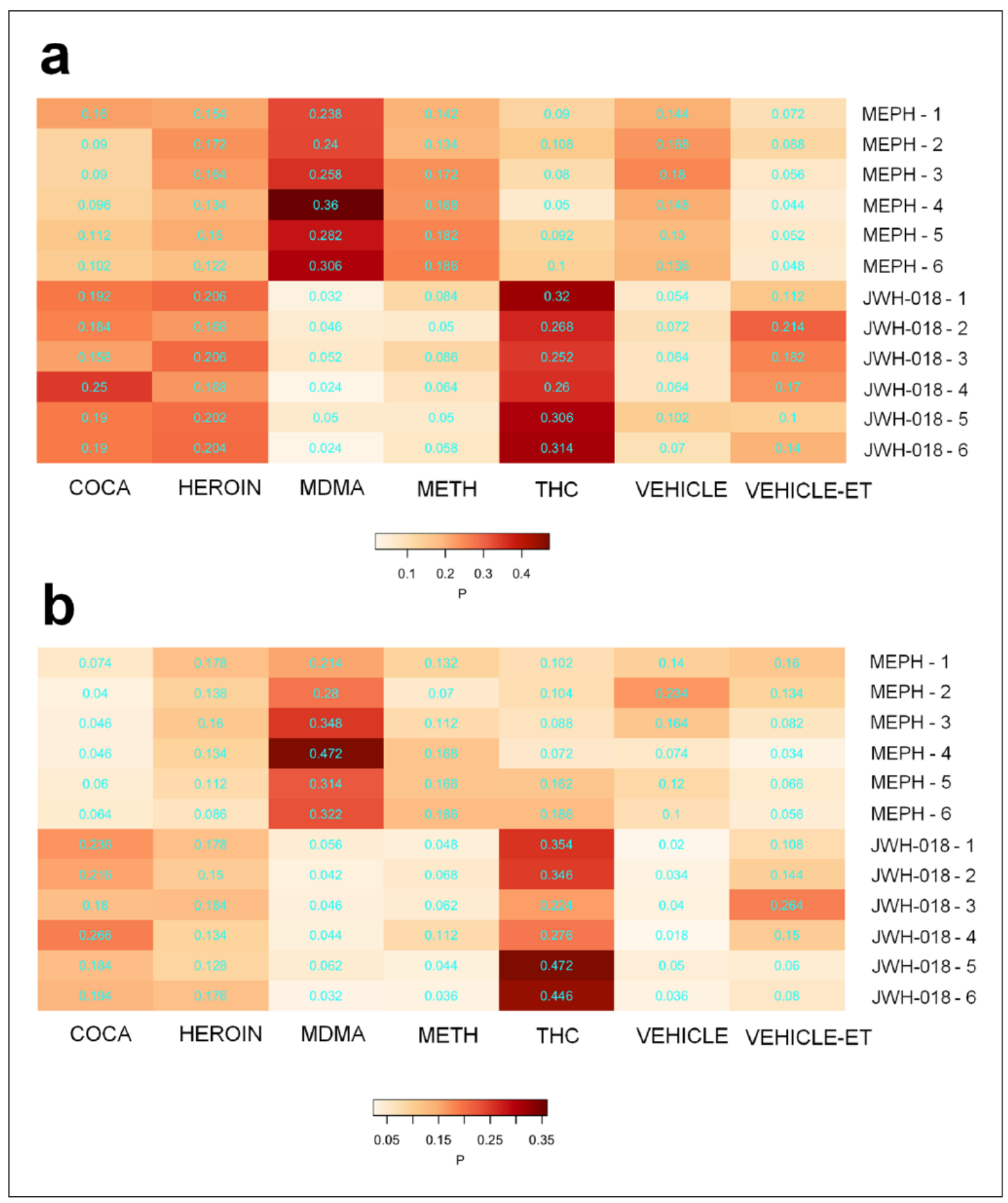

Figure 2. A machine learning algorithm model that predicts NPS as the closest traditional drugs (used to train the model). (a) The random forest classifier was trained with individual data $(n=6)$ for each classical drug. Each row corresponds to a sample administered with NPS on which the classification algorithm has been applied; each column is one of the possible classes to be predicted that had been used to train the model. The colour code goes from white (low probability of prediction) to red (high probability) in a gradual manner. The probability value, printed in cyan, corresponds to the fraction of votes of the trees in the ensemble. (b) Same as for part (a) but including only biomarkers from brain and plasma $1 \mathrm{~h}$ post administration.

compared with the vehicle (Figure 1(a), Supplementary Table 1, and Supplementary Table 6). Moreover, MDMA, a drug known to induce an acute depletion of serotonin in the brain, produced a relevant reduction of serotonin and its main metabolite, 5-HIAA, in all the investigated brain areas (Figure 1(a) and Supplementary Figure 1).
As proof of concept, we assayed mephedrone and JWH-018, two known NPS. These substances were selected because both had been recently included in the controlled substances schedule (Anon, 2010; Drug Enforcement Administration, 2013a, 2013b) given their similar pharmacological behaviour with illicit drugs of abuse (Papaseit et al., 2016; Theunissen et al., 2017; Toennes et al., 
2003). Furthermore, these substances were extensively studied in the literature, and their pharmacology was previously known (Papaseit et al., 2016; Theunissen et al., 2017), facilitating the confirmation of the prediction. The doses administered $(30 \mathrm{mg} / \mathrm{kg}$ for MEPH and $10 \mathrm{mg} / \mathrm{kg}$ for JWH-018) were selected from the literature (Marshell et al., 2014; Martínez-Clemente et al., 2013), and they were high and equivalent to the ones used for classical drugs of abuse. As expected from the literature, mephedrone was predicted to be an MDMA-like drug (Figure 2). In fact, both drugs presented a similar metabolomics fingerprint and were close in the PCA (Figure 1), concurring with the model prediction (Figure 2). The slight differences observed on the metabolomics fingerprint between MDMA and mephedrone (Figure 1) could be explained by the pharmacokinetic behaviour of mephedrone, which is described to have a shorter half-life when compared to MDMA (Martínez-Clemente et al., 2013; Papaseit et al., 2016). The prediction was correct in accordance to the mechanism of action of both drugs described in vitro (Baumann et al., 2012; Martínez-Clemente et al., 2012), the subjective effects described by users (Freeman et al., 2012) and their cardiovascular effects in humans (de la Torre et al., 2004; Papaseit et al., 2016). Actually, in 2009, mephedrone was consumed as a legal substitute to MDMA (Brunt et al., 2011). In a similar manner, JWH-018 was predicted to be a $\Delta^{9}$-THC-like compound, as demonstrated by comparable metabolomics fingerprints and their closeness in the PCA (Figures 1 and 2). This concurs with the drug's pharmacology that is primarily related to stimulation of the endocannabinoids receptors $\mathrm{CB}_{1}$ and $\mathrm{CB}_{2}$ (Atwood et al., 2010; Fattore and Fratta 2011; Maldonado et al., 2011). Moreover, many drug users prefer JWH-018 as a legal substitute for marijuana because of its marijuana-like effects (Baumann et al., 2014; Winstock and Barratt, 2013).

We further optimized the model by building a reduced one, without any substantial loss in accuracy or power in predicting NPS. Several combinations of metabolite blocks of variables were tested. It was observed that including only variables from $1 \mathrm{~h}$ drug treatment led to $100 \%$ accuracy and predicted all mephedroneand JWH-018-treated samples as MDMA and THC, respectively (Figure 2(b)). This simplified model could significantly reduce costs in the classification of new NPS. In parallel, we checked whether there were any overfitting problems due to the considerable number of metabolites used. If this were certain, it would be observed that while accuracy increased by including more variables as assessed by the LOOCV, the ability to predict NPS would decrease. To test this, we built 168 different models adding metabolites one by one. Again, a stable $100 \%$ accuracy was achieved by including 20 (or more) metabolites from all the targeted ones. As regards the ability to correctly predict NPS, using the 66 best predictors was enough to get a good prediction, with no sign of overfitting-related issues (Supplementary Figure 1 and Supplementary Table 8).

Certainly the addition of new metabolomics pathways (such as endocannabinoids and polar neurotransmitters) and the clinical monitoring of the rats (e.g. hear-rate, activity levels, temperature, etc.) would improve the precision of the model and its prediction between similar substances. We used a limited number of drugs as proof-of-principle to demonstrate the feasibility of the approach. Futures studies need to be performed using more drugs in order to correctly classify a wider range of NPS. For instance, the inclusion of other drug types such as LSD or ketamine would be required for expanding the model to the prediction of dissociative and hallucinogenic NPS. The success of our approach relies partly on ample knowledge of the pharmacology of classical drugs and selected NPS. Thus we could ensure that the doses assayed were pharmacologically active and equivalent. In the case of a completely unknown NPS entering the market, a dose-response study would be required to characterize its metabolomics fingerprint to allow pharmacological prediction through the model. In all, further studies targeting more drugs, more metabolic pathways and more administration times would be essential for the full development of the model. Additionally, future studies should take into consideration for the evaluation of the combination of different drugs.

The study of the drug mechanism of action (with in vitro approaches) and the drug-type characterization (with in silico strategies) are powerful techniques used in many scientific fields (i.e. drug discovery). However, missing gaps in the drugs' effects onset and the formation of bioactive metabolites could bias the pharmacology output. Our model combines experimental data (in vivo) and machine learning algorithms (in silico), which presents a step forward in the drug-pharmacological evaluation of novel substances, as this combination permitted the prediction of the pharmacological effects.

In summary, we developed a metabolomics model that classifies new drugs according to their pharmacological similarity with classical drugs of abuse. Our work is proposed as a fast and costeffective pharmacological tool, capable of evaluating the pharmacological profile of NPS. Our method has the potential to benefit risk evaluation policies by facilitating a rapid drug-type classification while reducing possible harm for the public related to the abuse of these emerging drugs.

\section{Authors note}

Marcel-lí Carbó is also affiliated to Department of Pharmacology, Toxicology and Therapeutic Chemistry, Faculty of Pharmacy and Food Sciences, University of Barcelona, Barcelona, Spain.

\section{Acknowledgements}

The authors are very grateful to Rafael Maldonado and Andrés Ozaita for their valuable assistance during $\Delta^{9}$-THC experiments. The valuable involvement of Mariona Cabanas and Josep Rodríguez-Morató is also acknowledged.

\section{Author contribution}

R.dT., O.J.P. J.R., T.B., M.C. and M.F conceived the idea and the funding for the project development. P.R., C.F., R.dT., O.P. and E.O. designed the animal experiments. R.dT., O.P. and E.O. created the metabolomics experimental procedure, and I.DT., M.D., R.dT. and O.P. designed the modelling procedure. E.O., P.R. and C.F. performed experiments in animals, and E.O., O.P. and R.dT. conducted the metabolomics experiments. I.DT. and M.D. performed the PCA and developed the predictive models. E.O., I.D.T., R.dT. and O.J.P. wrote the manuscript. All authors contributed to the editing of the paper.

\section{Declaration of conflicting interests}

The authors declare no potential conflicts of interest with respect to the research, authorship, and/or publication of this article.

\section{Funding}

The authors disclosed receipt of the following financial support for the research, authorship, and/or publication of this article: This work was supported by the European Commission (Drugs Policy Initiatives, Justice Programme 2014-2020, contract nº. HOME/2014/JDRU/AG/DRUG/7082, 
Predicting Risk of Emerging Drugs with In silico and Clinical Toxicology). Support by the Red de Trastornos Adictivos RTA RD12/0028/0006 and the DIUE of the Generalitat de Catalunya (2014 SGR 680; SGR 2014/1125) and the Program of Excellence Severo Ochoa (SEV-2012-0208) is also acknowledged. The Spanish Health National System is acknowledged for the contract of O.J.P. (CPII16/00027). This work was supported by a grant (I.D.T.) from the Impulse post-doctoral Marie-Curie co-funded program from CRG.

\section{Supplemental material}

Supplemental material for this article is available online.

\section{ORCID iD}

Eulàlia Olesti (iD) https://orcid.org/0000-0001-8677-9964

\section{References}

Anon (2010) European Comission, Press Release Database. Commission achieves EU-wide ban on ecstasy-like drug mephedrone. Available at: http://europa.eu/rapid/press-release_MEMO-10-646_en.htm

Atwood BK, Huffman J, Straiker A, et al. (2010) JWH018, a common constituent of "Spice" herbal blends, is a potent and efficacious cannabinoid CB 1 receptor agonist. Br J Pharmacol 160: 585-593.

Baumann MH, Ayestas MA Jr, Partilla JS, et al. (2012) The designer methcathinone analogs, mephedrone and methylone, are substrates for monoamine transporters in brain tissue. Neuropsychopharmacology 37: 1192-1203.

Baumann MH, Glennon RA and Wiley JL (2017) Current topics in behavioral neurosciences. In: Neuropharmacology of New Psychoactive Substances (NPS). Baltimore, MD: National Institute on Drug Abuse.

Baumann MH, Solis E Jr, Watterson LR, et al. (2014) Bath salts, spice, and related designer drugs: The science behind the headlines. J Neurosci 34: 15150-15158.

Bloomfield MA, Ashok AH, Volkow ND, et al. (2016) The effects of $\Delta 9$-tetrahydrocannabinol on the dopamine system. Nature 539: 369-377.

Breiman L (2001) Random forests. Machine Learning 45: 5-32.

Brunt TM, Poortman A, Niesink RJ, et al. (2011) Instability of the ecstasy market and a new kid on the block: Mephedrone. J Psychopharmacol 25: $1543-1547$.

Cai HL, Zhu RH and Li HD (2010) Determination of dansylated monoamine and amino acid neurotransmitters and their metabolites in human plasma by liquid chromatography-electrospray ionization tandem mass spectrometry. Anal Biochem 396: 103-111.

de la Torre R, Farré M, Roset PN, et al. (2004) Human pharmacology of MDMA. Ther Drug Monit 26: 137-144.

Drug Enforcement Administration D (2013a) 4-Methylmethcathinone (Mephedrone), Office of Diversion Control. Drug \& Chemical Evaluation Section p4. Springfield, VA: Office of Diversion Control, Drug Enforcement Administration.

Drug Enforcement Administration (2013b) JWH-018 [Synthetic Cannabinoid in Herbal Products], Office of Diversion Control. Drug \& Chemical Evaluation Section p1. Springfield, VA: Office of Diversion Control, Drug Enforcement Administration.

Fattore L (2016) Synthetic cannabinoids - further evidence supporting the relationship between cannabinoids and psychosis. Biol Psychiatry 79: 539-548.

Fattore L and Fratta W (2011) BeyondTHC: The new generation of cannabinoid designer drugs. Front Behav Neurosci 5: 60.

Fleckenstein AE, Beyeler ML, Jackson JC, et al. (1997) Methamphetamine-induced decrease in tryptophan hydroxylase activity: Role of 5-hydroxytryptaminergic transporters. Eur J Pharmacol 324: $179-186$.

Freeman TP, Morgan CJ, Vaughn-Jones J, et al. (2012) Cognitive and subjective effects of mephedrone and factors influencing use of a "new legal high." Addiction 107: 792-800.
Kroeze WK, Sassano MF, Huang XP, et al. (2015) PRESTO-Tango as an open-source resource for interrogation of the druggable human GPCRome. Nat Struct Mol Biol 22: 362-369.

Lu H, Yu J, Wang J, et al. (2016) Simultaneous quantification of neuroactive dopamine serotonin and kynurenine pathway metabolites in gender-specific youth urine by ultra performance liquid chromatography tandem high resolution mass spectrometry. J Pharm Biomed Anal 122: 42-51.

Madras BK (2017) The growing problem of new psychoactive substances (NPS). Curr Top Behav Neurosci 32: 1-18.

Maldonado R, Berrendero F, Ozaita A, et al. (2011) Neurochemical basis of cannabis addiction. Neuroscience 181: 1-17.

Marcos J, Renau N, Casals G, et al. (2014) Investigation of endogenous corticosteroids profiles in human urine based on liquid chromatography tandem mass spectrometry. Analytica Chimica Acta 812: 92-104.

Marcos J, Renau N, Valverde O, et al. (2016) Targeting tryptophan and tyrosine metabolism by liquid chromatography tandem mass spectrometry. J Chromatogr A 1434: 91-101.

Marshell R, Kearney-Ramos T, Brents LK, et al. (2014) In vivo effects of synthetic cannabinoids JWH-018 and JWH-073 and phytocannabinoid $\Delta 9$-THC in mice: Inhalation versus intraperitoneal injection. Pharmacol Biochem Behav 124: 40-47.

Martínez-Clemente J, Escubedo E, Pubill D, et al. (2012) Interaction of mephedrone with dopamine and serotonin targets in rats. Eur Neuropsychopharmacol 22: 231-236.

Martínez-Clemente J, López-Arnau R, Carbó M, et al. (2013) Mephedrone pharmacokinetics after intravenous and oral administration in rats: Relation to pharmacodynamics. Psychopharmacology (Berl) 229: 295-306.

Meinhardt MW, Sévin DC, Klee ML, et al. (2015) The neurometabolic fingerprint of excessive alcohol drinking. Neuropsychopharmacology 40: 1259-1268.

Miliano C, Serpelloni G, Rimondo C, et al. (2016) Neuropharmacology of new psychoactive substances (NPS): Focus on the rewarding and reinforcing properties of cannabimimetics and amphetamine-like stimulants. Front Neurosci 10: 153.

Näsström E, Vu Thieu NT, Dongol S, et al. (2014) Salmonella Typhi and Salmonella Paratyphi A elaborate distinct systemic metabolite signatures during enteric fever. Elife 3: 1-19.

Papaseit E, Olesti E, de la Torre R, et al. (2017) Mephedrone concentrations in cases of clinical intoxication. Curr Pharm Des 23: 55115522.

Papaseit E, Pérez-Mañá C, Mateus JA, et al. (2016) Human pharmacology of mephedrone in comparison to MDMA. Neuropsychopharmacology 41: 2704-2713.

Simmler LD, Buser TA, Donzelli M, et al. (2013) Pharmacological characterization of designer cathinones in vitro. Br J Pharmacol 168: 458-470.

Stone D, Stahl DC, Hanson GR, et al. (1986) The effects of 3,4-methylenedioxymethamphetamine (MDMA) and 3,4-methylenedioxyamphetamine (MDA) on monoaminergic systems in the rat brain. Eur $J$ Pharmacol 128(1-2): 41-48.

Strachan RT, Ferrara G and Roth BL (2006) Screening the receptorome: An efficient approach for drug discovery and target validation. Drug Discov Today 11: 708-716.

Theunissen EL, Hutten NRPW, Mason NL, et al. (2017) Neurocognition and subjective experience following acute doses of the synthetic cannabinoid JWH-018: A phase 1, placebo-controlled, pilot study. Br J Pharmacol. Epub ahead of print 29 November 2017. DOI: 10.1111/bph.14066.

Toennes SW, Harder S, Schramm M, et al. (2003) Pharmacokinetics of cathinone, cathine and norephedrine after the chewing of khat leaves. Br J Clin Pharmacol 56: 125-130.

United Nations Office on Drugs and Crime (2017) Global overview of drug demand and supply. Herndon, VA: United Nations Publication, pp. 3-65.

Winstock AR and Barratt MJ (2013) Synthetic cannabis: A comparison of patterns of use and effect profile with natural cannabis in a large global sample. Drug Alcohol Depend 131: 106-111.

Wishart DS (2016) Emerging applications of metabolomics in drug discovery and precision medicine. Nat Rev Drug Discov 15: 473-484. 